\title{
Searching for anatomical correlates of olfactory lateralization in the honeybee antennal lobes: A morphological and behavioural study
}

\author{
Elisa Rigosi $i^{a, b}$, Elisa Frasnellic ${ }^{c}$, Claudio Vinegoni ${ }^{d}$, Renzo Antolini ${ }^{\mathrm{e}}$, Gianfranco Anfora ${ }^{\mathrm{a}}$, \\ Giorgio Vallortigara ${ }^{b}$, and Albrecht Haase ${ }^{e}$ \\ a IASMA Research and Innovation Center, Fondazione E. Mach, Via E. Mach 1, 38010, San \\ Michele all'Adige (TN), Italy \\ b $\mathrm{CIMeC}$, Centre for Mind/Brain Sciences, University of Trento, Corso Bettini 31, 38068 Rovereto \\ (TN), Italy \\ ${ }^{c}$ Konrad Lorenz Institute for Evolution and Cognition Research, Adolf Lorenz Gasse 2 A-3422 \\ Altenberg, Austria \\ d Center for Systems Biology and Center for Molecular Imaging Research, Massachusetts \\ General Hospital, Harvard Medical School, 185 Cambridge Street, Boston, Massachusetts 02114, \\ USA \\ e Physics Department, University of Trento, Via Sommarive 14, 38050 Povo (TN), Italy
}

\section{Abstract}

The honeybee, Apis mellifera L. (Hymenoptera: Apidae), has recently become a model for studying brain asymmetry among invertebrates. A strong lateralization favouring the right antenna was discovered in odour learning and short-term memory recall experiments, and a lateral shift favouring the left antenna for long-term memory recall. Corresponding morphological asymmetries have been found in the distribution of olfactory sensilla between the antennae and confirmed by electrophysiological odour response measurements in isolated right and left antennae. The aim of this study was to investigate whether a morphological asymmetry can be observed in the volume of the primary olfactory centres of the central nervous system, the antennal lobes (ALs). Precise volume measurements of a subset of their functional units, the glomeruli, were performed in both sides of the brain, exploiting the advantages of two-photon microscopy. This novel method allowed minimal invasive acquisition of volume images of the ALs, avoiding artefacts from brain extraction and dehydration. The study was completed by a series of behavioural experiments in which response asymmetry in odour recall following proboscis extension reflex conditioning was assessed for odours, chosen to stimulate strong activity in the same glomeruli as in the morphological study. The volumetric measurements found no evidence of lateralization in the investigated glomeruli within the experimental limits. Instead, in the behavioural experiments, a striking odour dependence of the lateralization was observed. The results are discussed on the basis of recent neurophysiological and ethological experiments in $A$. mellifera.

(C) 2011 Elsevier B.V. All rights reserved.

Correspondence to: Elisa Rigosi.

Publisher's Disclaimer: This is a PDF file of an unedited manuscript that has been accepted for publication. As a service to our customers we are providing this early version of the manuscript. The manuscript will undergo copyediting, typesetting, and review of the resulting proof before it is published in its final citable form. Please note that during the production process errors may be discovered which could affect the content, and all legal disclaimers that apply to the journal pertain. 


\section{Keywords}

Apis mellifera; brain asymmetry; olfactory learning; antennal lobes; two-photon microscopy

\section{Introduction}

The olfactory pathway in the honeybee, Apis mellifera L. (Hymenoptera: Apidae) is a wellknown model of coding, storing, and recalling information. At the very periphery, the level of the antennae, odour stimuli are detected by olfactory receptor neurons (ORNs) housed in olfactory sensilla. The ORNs' axons run via the antennal nerves to the first olfactory information processing centres of the insect brain, the antennal lobes (ALs). Right and left $\mathrm{AL}$ are bilaterally symmetrical structures formed by $\sim 160$ subunits, so called glomeruli, each supposed to be invaded by only one to two specific olfactory receptor classes. The glomeruli are linked by $\sim 4000$ local interneurons (LNs). Each odour elicits a specific spatio-temporal pattern of glomerular activation coming from the ORNs potentials [12]. In each glomerular neuropil, the peripheral olfactory signal undergoes subsequent modulation due to LNs and to descending centrifugal neurons from the deuterocerebrum. The final output, is a speciesspecific fine tuned combinatorial coding pattern forwarded by $\sim 800$ projection neurons (PNs) to higher order brain areas (reviewed in [14]). Several anatomical and functional atlases of the honeybee ALs have been created in the past years [8], [11], [12], [26]. They allow individual glomerular identification through ALs arrangements and the single glomerular role in the ALs odour response maps.

Morphological changes in the neuropil of single glomeruli have been revealed as an effect of olfactory learning. In particular, experience-dependent changes in the glomerular volume have been shown to take place during the bee's lifetime and to be highly specific to some glomeruli [27], [28], [3], [4]. Recently, a striking volume increase after single odour learning tasks has been described [17]. Bees, conditioned to extend their proboscis in response to a particular odour stimulus using the so called proboscis extension reflex paradigm (PER) [2], showed significant volume increase of specific glomeruli linked to the positive performance in learning retention tests 3 days after odour training.

The PER paradigm has been widely used as a behavioural learning method over the years, and it has been recently applied to describe a form of lateralization in honeybee olfactory learning. When conditioned using PER, bees showed better learning with only their right rather than only their left antenna in use [22], [9], [1]. Morphological analyses found a significantly higher number of olfactory sensilla on the right antenna [22], [9]. However, lateralization of olfactory learning in bees is unlikely to be explained by morphological asymmetries in the antennae only, for experiments have shown, that after training with both antennae in recall tests $1-2 \mathrm{~h}$ after conditioning bees performed better with only their right antenna instead of only their left antenna in use but $6 \mathrm{~h}$ after training the memory had performed a lateral shift, being now better retrieved by the left instead of the right antenna [25]. Possible anatomical asymmetries within the brain have not been systematically investigated so far. A single rough comparison in [28] has not shown any symmetry breaking.

In order to improve our understanding of the olfactory lateralization in honeybees, in this study we precisely measured the volume of specific glomeruli in left and right AL in foraging bees and we replicated the learning PER paradigm with odours that evoke activity in these specific glomeruli. 


\section{Materials and methods}

\subsection{Insects}

Italian forager honeybees, A. mellifera ligustica Spin., were collected during summer 2009 and 2010 in San Michele all'Adige and Mattarello (Trento, Italy).

\subsection{Optical Imaging}

For the imaging studies of the antennal lobes, bees $(N=12)$ have been prepared in accordance to a well-established protocol [13]. After chilling until immobility, insects were placed into custom made imaging stages and held in place using soft melting wax (Kerr, Sybron Dental Specialties). To expose the antennal lobes, a window was cut into the cuticle, and glands and trachea were gently removed. The neural sheath was digested by immersion in a $1 \%$ solution of Protease Type XIV (Sigma-Aldrich) for $5 \mathrm{~min}$ at $\sim 40^{\circ} \mathrm{C}$. The bee brain was then stained by bath-application of a 50 $\mu \mathrm{M}$ solution of the membrane-selective dye RH795 (Invitrogen) for $3 \mathrm{~h}$. After rinsing with Ringer's solution, the bees were ready to be imaged.

Antennal lobes were volumetrically imaged without extracting the brain in order to prevent artefacts due to tissue isolation, fixation, and dehydration [5]. This was realised using twophoton microscopy [6], [29] which offers enhanced penetration depth and a higher axial resolution than conventional fluorescence microscopy and which was recently demonstrated for whole antennal lobe imaging[15]. In this experiment a two-photon microscope (Ultima IV, Prairie Technologies) was used in combination with an ultra-short pulsed laser (Mai Tai Deep See HP, Spectra-Physics) as excitation source, tuned to the wavelength of $1040 \mathrm{~nm}$, corresponding to the maximum of the dye's two-photon cross section within our tuning range. The beam was focused on the sample with a water immersion objective (Olympus, $40 \mathrm{x}, \mathrm{NA}=0.8$ ), which provides a field of view of approximately $300 \mu \mathrm{m}$. The system's resolution was measured to be diffraction limited, resulting in a point spread function of Gaussian width $\sigma_{\mathrm{x}, \mathrm{y}}=230 \mathrm{~nm}$ transversally and $\sigma_{\mathrm{z}}=1.1 \mu \mathrm{m}$ axially. The dye's fluorescence is epicollected by the same objective, separated from the backscattered excitation light with a dichroic beam-splitter, filtered by a $70 \mathrm{~nm}$ bandpass filter centred at around $525 \mathrm{~nm}$ (both Chroma Technology), and finally detected by a photomultiplier tube (Hamamatsu Photonics). Average laser powers were around $10 \mathrm{~mW}$ on the sample.

Volumetric measurements were obtained by collecting stacks of AL image slices by varying the focal plane in steps of $3 \mu \mathrm{m}$ along the antero-posterior axis. The imaging depth was mostly limited by the diffusion depth of the bath-applied dye, and was found to be around $150 \mu \mathrm{m}$.

For the subset of glomeruli to analyse those were chosen which firstly are most strongly involved in learning processes for common plant odours and which secondly show very diverse activation pattern in functional imaging studies in order to look for a possible odour specificity of the results. This should help focussing onto learning-associated lateralization effects and to magnify a possible odour-dependence. The first aspect was evaluated following a previous morphological study of Hourcade et al. in 2009 [17]. We selected Glomerulus T1-17 showing significant increased volume in both odour conditioning experiments in [17], and T1-33 and T1-48 both significantly increased in one out of the two tests. We added Glomeruli T1-28 and T1-42 to our subset for their opposing odour response maps, the first rather broadband and the second rather sharp [12] with a strongest response to e.g. 2-octanone, one of the substances used in our behavioural tests.

Image segmentation for the volumetric reconstruction was performed using the software Amira (Visage Imaging). A semi-automatic protocol was defined, where single glomeruli 
were traced in the principal planes using the watershedding method "magic wand". Then the volume images were reconstructed by the program's wrapping interpolation method.

The robustness of this method was checked by slight variation of initial parameters in the reconstruction procedure. If the outcome varied too strongly, the image quality was classified insufficient and the data were discarded. This was the case mostly due to poor dye diffusion or shadows from the remaining trachea. Because the absolute volumes of the single glomeruli were fluctuating among different individuals, the data of left and right side were directly compared for each bee, quantifying the left-right asymmetry by the lateralization index $L=V_{\mathrm{R}} /\left(V_{\mathrm{R}}+V_{\mathrm{L}}\right)$, ranging from 0 to 1 around the symmetry point 0.5 , where $V_{\mathrm{R}}$ and $V_{\mathrm{L}}$ denote the right and left volume, respectively. Glomerular volumes were analyzed by analysis of variance (ANOVA) with glomerular type and side as within-subjects factors.

\subsection{Behaviour}

Bees were cooled in $750 \mathrm{ml}$ containers until immobilised and secured in holders [2], [25]. They were then assigned randomly to groups for the occlusion of one antenna, and $1 \mathrm{~h}$ later all bees were trained in the same way. The experiment was carried out on three groups of bee referring to the antenna in use. The bees in one group ( $N=65$ honey bees) had their left antenna coated with a silicone compound (Silagum-Mono, DMG), those in the second group $(N=63)$ had their right antenna coated, while both antennae of the bees in the third group were left uncoated $(N=66)$. Each group of bees was subdivided into three training groups on the basis of the odour compound used as a conditioned stimulus

One hour after the antennae had been coated each bee was placed in front of an exhaust fan and trained using as positive conditioned stimulus (CS+) the odour compounds 1-octanol ( $N=25$ both antennae (CTRL); $N=23$ only right antenna in use (RA); $N=22$ only left antenna in use (LA)), 2-octanone ( $N=19$, CTRL; $N=22$, RA; $N=20$, LA) (both Fluka, purity $>95 \%$ ), or (-)-linalool ( $N=22$, CTRL; $N=18, \mathrm{RA} ; N=23, \mathrm{LA})$ (Sigma-Aldrich, $>98.5 \%$ purity) together with $1 \mathrm{M}$ sucrose solution as a food reward (unconditioned stimulus, US).

By electing these specific odours, we connected the behavioural tests to our optical study, since these odours stimulate a very diverse response pattern in the measured glomerular subset of the AL [12], [15], [23] and seem therefore good candidates to manifest a possible odour dependence of the test results.

$10 \mu$ of each odour compound were dissolved in $3 \mathrm{ml}$ of the sucrose solution. The negative stimulus was a saturated saline solution ( $\left.\mathrm{CS}^{-}\right) .3$ trials were performed spaced $6 \mathrm{~min}$ apart. In the first trial a droplet of the CS+/US solution at the end of a 23 gauge needle was held $1 \mathrm{~cm}$ above the bee's antennae, after $5 \mathrm{~s}$ the antennae were touched, which led to PER. The bee was then allowed to ingest the drop of the odour-sugar solution. The procedure was repeated with the saline solution, which did not trigger PER but avoidance by moving the antennae away from the droplet. In trial 2 and 3 the procedure of trial 1 was repeated. Usually in trial 3 the CS+/US stimulus triggered PER without the need of touching the antennae.

Retention was tested $1 \mathrm{~h}$ later by presenting the odour dissolved in distilled water or the saline solution and holding the droplet $1 \mathrm{~cm}$ from the antennae while moving it slightly without touching the antennae. These CS+ and CS- solutions were presented for 5s. Each bee was tested a total of 10 paired trials, where CS+ and CS- were presented in a random order with 60 s between every odour presentation. We recorded every time the bee extended the proboscis. The percentages of success were scored as extensions of the proboscis to odours and no extension to saturated salt solution (PER(CS+)-PER(CS-))/(\# paired trials). 
As the measurements do not meet the normality assumption of an ANOVA, data of each odour were analyzed by Kruskal-Wallis one-way analysis of variance, with the 3 different experimental conditions (left antenna/right antenna/both antennae in use) as independent groups. Within the groups of different antennae in use, performance in the various odour tests was analyzed by Kruskal-Wallis one-way analysis of variance with the 3 different odour compounds as independent groups. When a statistically difference was found, a Mann-Whitney $U$ test was used for looking at the differences between the means of all groups.

\section{Results}

The optical imaging experiment allowed determination of the volume of single glomeruli (Fig. 3a). Measured absolute volumes were about twice the size of those found in previous studies [28], [17] where dehydrated samples had been used. This shows the order of magnitude of the shrinkage effects avoided here. From the reconstructed volume images we determined the relative volume asymmetry between left and right AL. For the five glomeruli that have been chosen, the mean values and the standard errors of the lateralization index $(N=12)$ are shown in Figure 3b: for T1-17: 0.51 \pm 0.01 , for T1-28: 0.49 \pm 0.02 , for T1-33: $0.50 \pm 0.01$, for T1-42: $0.50 \pm 0.01$, and for T1-48: $0.49 \pm 0.01$. Analysis of variance revealed a significant difference in volume among different glomerular types $\left(F_{4,44}=117.27, p<0.001\right)$ (Fig. 3a), but no significant glomerular volume size differences between sides $\left(F_{1,11}=0.26\right.$, $p=0.617$ ). No significant effect of the interaction between glomerular type and side was found $\left(F_{4,44}=0.83, p=0.51\right)$.

The results of the behavioural tests are shown in Figure 4. The analysis of variance, using the Kruskal-Wallis test, revealed no significant differences in recall tests among the three groups of bees for 1-octanol $\left(\chi^{2}=1.02, p=0.60, N=70\right)$ and 2-octanone $\left(\chi^{2}=0.97, p=0.62\right.$, $N=61$ ), bees showed no differences in recall test either with only their right antenna in use or with only their left or with both antenna in use. In contrast, bees trained with (-)-linalool showed a significant effect of the antenna in use $\left(\chi^{2}=9.91, p<0.01, N=63\right)$. The MannWhitney $U$ test revealed a significant difference between bees trained with both antennae in use and individuals with only their left antenna in use $(U=128.5, p<0.01, N=45)$. A similar difference was found comparing bees with their right antenna in use and bees with their left antenna in use ( $U=112.5, p<0.05, N=41)$. No differences were found between bees using both antennae and bees using only their right antenna ( $U=190.0, p=0.83, N=40)$.

The Kruskal-Wallis test revealed significant odour effect in the performance of bees trained only with the left antenna in use $\left(\chi^{2}=6.17, p<0.05, N=65\right)$; Mann-Whitney $U$ test performed inside the left antenna's group, showed significant differences both between (-)-linalool and 1-octanol $(U=160.0, p<0.05, N=45)$ and between ( - -linalool and 2-octanone ( $U=143.0$, $p<0.05, N=43)$. No difference was found between 1-octanol and 2-octanone ( $U=215.5$, $p=0.91, N=42$ ). Within the groups with only the right or with both antennae in use the Kruskal-Wallis test revealed no significant differences among different odour compounds: $\left(\chi^{2}=0.41, p=0.81, N=63\right)$ and $\left(\chi^{2}=0.20, p=0.90, N=66\right)$, respectively.

\section{Discussion}

The two-photon imaging experiments allowed for the first time precise volume measurements in the honeybee antennal lobe, without extraction and fixation of the brain. This improved the spatial resolution by almost an order of magnitude with respect to the only previously published work comparing precisely the AL morphology in the two brain hemispheres [28], based on histological slices at distances of $25 \mu \mathrm{m}$. Moreover, it avoided artefacts due to anisometric shrinkage and diffraction-index mismatch [5] occurring during 
fixation and clearing in experiments on extracted brains which are usually used for morphological imaging of the bee brain [11], [17]. The measurement accuracy is limited by the decreasing contrast at higher imaging depth, causing problems in defining precisely the border between single glomeruli.

Within this newly established accuracy limits, our study showed that the chosen subset of glomeruli does not differ in volume between the right and left side of the brain in honeybee foragers without controlled experience, which confirms the previous study [28].

At the behavioural level, previous investigations on olfactory lateralization in bees with unknown experience showed a significant lateralization towards the right antenna (i.e. higher percentage of success in PER $1 \mathrm{~h}$ after conditioning by the right antenna [22], [9]), as well as in peripheral detection of odorants measured with electroantennography [1]. Moreover olfactory sensilla showed to be higher in number on the right antenna rather than on the left [9]. This peripheral lateralization, found in bees without controlled experience, did not show a correspondence in the morphology of the measured set of glomeruli or, alternatively, we were not able to detect it under our experimental conditions. Even focusing on the mere anatomical view, it might be likely that the distinctly different number of olfactory sensilla between the antennae could not be sufficient by themselves to cause consequent volume impairment in the antennal lobe glomeruli, since the connection between sensilla and AL activation pattern is highly nonlinear [18].

Moreover, with changing behavioural tasks and foraging experiences antennal lobes and specific glomeruli undergo a significant volume size modification that is age and odourexposure dependent [28], [27]. Such a volume plasticity of first olfactory centres has been also described in other species [7], [16]. For this reason, it is likely that any significant difference in volumes between the ALs in bee foragers with no controlled experience might be hidden under bigger volume fluctuations of specific glomeruli, influenced by both shortterm and long-term odour experience. In addition, it has to be noted that at the behavioural level, olfactory asymmetry in bees was demonstrated to be dependent on the time interval between odour conditioning and odour retrieval [25]. In particular, short-term memory (STM) recall tests seem to be better performed with the right rather than with the left antenna, but starting from 6 hours after training, bees showed to better retain odours when they have only their left antenna in use compared with bees with only their right antenna in use, which might be associated to a laterally displaced long-term memory (LTM). Due to this lateral shift of unilateral memories (or the access to unilateral memories) we might observe a corresponding shift in neuronal modelling both in the ALs and in higher brain centres. In forager bees without controlled experience, the absence of lateralization in the glomerular volume might by due to these competing memory processes in the two sides of the brain occurring on different time scales. Only the amplification of one of these memory processes in controlled conditioning experiments will give a definite answer. Our results serve more as a baseline for future measurements, showing the volumetric symmetry being the long-term steady state.

To connect the behavioural STM recall tests to our optical study of a subset of glomeruli in the AL, we chose to test odour compounds which, in a previous morphological imaging study [17], induced in bees the highest volumetric plasticity in the same glomerular subset and which, in a previous functional imaging studies [12], [15], [23], showed very diverse activity pattern in these glomeruli. This should help to detect possible odour dependence in the results. One form of odour dependence asymmetry has been demonstrated already in [10], based on retroactive interference between STM and LTM. Our behavioural experiments add a new aspect to these results, showing that in recall test $1 \mathrm{~h}$ after 
conditioning, different type of plant odour volatiles being able to drive asymmetries or not, depending on the biological relevance of the plant compound.

The elected odour stimuli were 1-octanol, an alcoholic compound, and 2-octanone, a ketone. For both these odours we found no significant differences in STM recall tests between animals trained with only their left antenna and those with only their right antenna in use. We then trained and tested another set of bees with (-)-linalool, a monoterpene floral compound, for which previous studies had shown a clear right asymmetry in odour learning recall tests [9] and our results confirmed the significant right-side dominance. These results suggest that STM induced lateralization in bees might be odour-specific, or that lateral shift [25] associated with the transition from STM to LTM occurs at different time scales for different types of odours. (-)-linalool is one of the most common derivates of floral scents playing a crucial role as cue for pollinators [19], [20]. It was demonstrated that honeybees were able to learn complex odour mixtures through a subset of key odours such as (-)linalool [24] and that (-)-linalool elicited higher levels of response when it was presented after conditioning to a mixture in respect to others components of the mixture [21]. Instead, 1-octanol, and 2-octanone are unspecific and ubiquitous volatiles released from the green organs of the plants and thus of minor importance in pollinator plant interaction. So this strikingly different biological relevance of the odour compounds might be a reason for the observed difference in lateralization.

Regarding a possibly different time scale for the lateral shift from STM to LTM, Rogers and Vallortigara [25] found the balance point between left and right side dominance in memory recall tests to be $3 \mathrm{~h}$ for lemon as odour stimulus. For the unspecific and ubiquitous volatiles tested here, this point might be shifted to shorter times causing the symmetric behaviour after $1 \mathrm{~h}$ which was observed by us. So an important next experimental step will be the extension of memory recall tests to different points in time to measure the time-course in the lateralized odour-storage for these compounds.

To deeper address this aspect, conditioning experiments with a large range of different odours are needed. At the same time, the volumetric comparison of left and right hemisphere antennal lobes has to be performed on conditioned bees at distinct times after conditioning to be able to better compare these data with the results from corresponding behavioural experiments [25].

\section{Acknowledgments}

This work has been realized also thanks to the support from the Provincia Autonoma di Trento and the Fondazione Cassa di Risparmio di Trento e Rovereto. C.V. acknowledges Provincia Autonoma di Trento (project COMNFI) and NIH grant RO1EB006432.

\section{References}

1. Anfora G, Frasnelli E, Maccagnani B, Rogers LJ, Vallortigara G. Behavioural and electrophysiological lateralization in a social (Apis mellifera) but not in a non-social (Osmia cornuta) species of bee. Behav Brain Res. 2010; 206:236-9.10.1016/j.bbr.2009.09.023 [PubMed: 19766143]

2. Bitterman ME, Menzel R, Fietz A, Schafer S. Classical conditioning of proboscis extension in honeybees (Apis mellifera). J Comp Psychol. 1983; 97:107-19.10.1037/0735-7036.97.2.107 [PubMed: 6872507]

3. Brown SM, Napper RM, Thompson CM, Mercer AR. Stereological Analysis Reveals Striking Differences in the Structural Plasticity of Two Readily Identifiable Glomeruli in the Antennal Lobes of the Adult Worker Honeybee. J Neurosci. 2002; 22(19):8514-22. [PubMed: 12351725] 
4. Brown SM, Napper RM, Mercer AR. Foraging Experience, Glomerulus Volume, and Synapse Number: A Stereological Study of the Honey Bee Antennal Lobe. J Neurobiol. 2004; 60(1):40-50. [PubMed: 15188271]

5. Bucher D, Scholz M, Stetter M, Obermayer K, Pfluger HJ. Correction methods for threedimensional reconstructions from confocal images: I. tissue shrinking and axial scaling. J Neurosci Methods. 2000; 100:135-143.10.1016/S0165-0270(00)00245-4 [PubMed: 11040376]

6. Denk W, Strickler JH, Webb WW. Two-photon laser scanning fluorescence microscopy. Science. 1990; 248(4951):73-6. [PubMed: 2321027]

7. Devaud JM, Acebes A, Ferrus A. Odor Exposure Causes Central Adaptation and Morphological Changes in Selected Olfactory Glomeruli in Drosophila. J Neurosci. 2001; 21(16):6274-82. [PubMed: 11487650]

8. Flanagan D, Mercer AR. An atlas and 3-D reconstruction of the antennal lobes in the worker honey bee, Apis mellifera L. (Hymenoptera: Apidae). J Insect Morphol Embryol. 1989; 18(2-3):145-159.

9. Frasnelli E, Anfora G, Trona F, Tessarolo F, Vallortigara G. Morpho-functional asymmetry of the olfactory receptors of the honeybee (Apis mellifera). Behav Brain Res. 2010; 209(2):221-5. [PubMed: 20138089]

10. Frasnelli E, Vallortigara G, Rogers LJ. Response competition associated with right-left antennal asymmetries of new and old olfactory memory traces in honeybees. Behav Brain Res. 2010; 209(1):36-41. [PubMed: 20085786]

11. Galizia CG, Mcllwarth SL, Menzel R. A digital three-dimensional atlas of the honeybee antennal lobe based on optical sections acquired by confocal microscopy. Cell Tissue Res. 1999; 295(3): 383-94. [PubMed: 10022959]

12. Galizia CG, Sachse S, Rappert A, Menzel R. The glomerular code for odor representation is species specific in the honeybee Apis mellifera. Nat Neurosci. 1999; 2:473-8. [PubMed: 10321253]

13. Galizia, CG.; Vetter, R. Optical methods for analyzing odor-evoked activity in the insect brain. In: Christensen, TA., editor. Advances in Insect Sensory Neuroscience. Boca Raton: CRC press; 2004. p. 349-92.

14. Galizia CG, Szyszka P. Olfactory coding in the insect brain: molecular receptive ranges, spatial and temporal coding. Entomol Exp Appl. 2008; 128(1):81-92.

15. Haase A, Rigosi E, Trona F, Anfora G, Vallortigara G, Antolini R, Vinegoni C. In-vivo twophoton imaging of the honey bee antennal lobe. Biomed Opt Express. 2011; 2:131-8. [PubMed: 21326643]

16. Harvey FE, Colley JJ. Effects of external chemical environment on the developing olfactory bulbs of the mouse (Mus musculus). Brain Res Bull. 1984; 13(4):541-7. [PubMed: 6525529]

17. Hourcade B, Perisse E, Devaud JM, Sandoz JC. Long-term memory shapes the primary olfactory center of an insect brain. Learn Mem. 2009; 16(10):607-15. [PubMed: 19794186]

18. Kelber C, Rössler W, Kleineidam CJ. Multiple olfactory receptor neurons and their axonal projections in the antennal lobe of the honeybee Apis mellifera. J Comp Neurol. 2006; 496:395405. [PubMed: 16566001]

19. Knudsen JT, Tollsten L, Bergström LG. Floral scents-a checklist of volatile compounds isolated by head-space techniques. Phytochemistry. 1993; 33(2):253-80.

20. Knudsen JT, Eriksson R, Gershenzon J, Ståhl B. Diversity and distribution of floral scent. Bot Rev. 2006; 72(1):1-120.

21. Laloi D, Bailez O, Blight M, Roger B, Pham-Delegue M-H, Wadhams LJ. Recognition of complex odors by restrained and free-flying honeybees, Apis mellifera. J Chem Ecol. 2000; 26:2307-19.

22. Letzkus P, Ribi WA, Wood JT, Zhu H, Zhang SW, Srinivasan MV. Lateralization of olfaction in the honeybee Apis mellifera. Curr Biol. 2006; 16:1471-6.10.1016/J.cub.2006.05.060 [PubMed: 16860748]

23. Peele P, Ditzen M, Menzel R, Galizia CG. Appetitive odor learning does not change olfactory coding in a subpopulation of honeybee antennal lobe neurons. J Comp Physiol A. 2006; 192:1083103.

24. Reinhardt J, Sinclair M, Srinivasan MV, Claudianos C. Honeybees Learn Odour Mixtures via a Selection of Key Odorants. PLoS One. 2010; 5(2):e9110. [PubMed: 20161714] 
25. Rogers LJ, Vallortigara G. From antenna to antenna: lateral shift of olfactory memory in honeybees. PLoS One. 2008; 2008(3):e2340.10.1371/journal.pone.0002340 [PubMed: 18523636]

26. Sachse S, Rappert A, Galizia CG. The spatial representation of chemical structures in the antennal lobe of honeybees: steps towards the olfactory code. Eur J Neurosci. 1999; 11(11):3970-82. [PubMed: 10583486]

27. Sigg D, Thompson CM, Mercer AR. Activity-Dependent Changes to the Brain and Behaviour of the Honey Bee, Apis mellifera (L. ) J Neurosci. 1997; 17(18):7148-56. [PubMed: 9278549]

28. Winnington AP, Napper RM, Mercer AR. Structural Plasticity of Identified glomeruli in the antennal Lobes of Adult Worker Honey Bee. J Comp Neurol. 1996; 365:479-90. [PubMed: 8822183]

29. Zipfel WZ, Williams RM, Webb WW. Nonlinear magic: multiphoton microscopy in the biosciences. Nat Biotechnol. 2003; 21:1369-77. [PubMed: 14595365] 


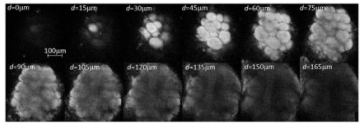

Fig. 1.

Stack of two-photon microscopy images of a left antennal lobe of Apis mellifera foragers. The tissue is bath-stained with membrane-selective RH795 dye. The field of view of the used $40 \mathrm{x}$ objective is $0.3 \mathrm{~mm}$. Total imaging depth is $165 \mu \mathrm{m}$. 


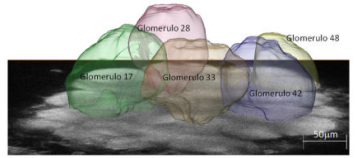

Fig. 2.

Single image of the left antennal lobe of Apis mellifera at an imaging depth of approximately $80 \mu \mathrm{m}$, superimposed with the reconstructed volume images of the analysed glomeruli. 


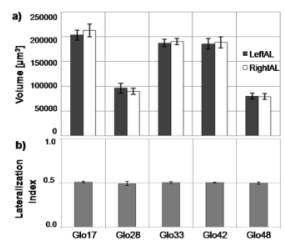

Fig. 3.

Right and left absolute volumes (a) and their correspondent lateralization index (b) of the 5 investigated T1 glomeruli of the honeybee antennal lobes. Mean values are shown together with their standard errors $(N=12)$. 


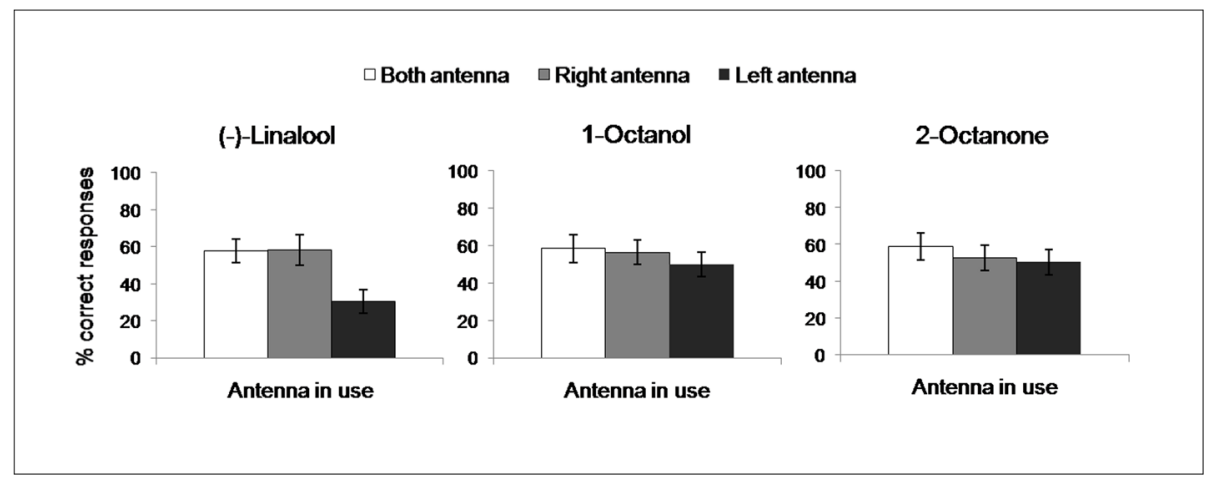

Fig. 4.

Mean \pm SEM of correct responses of Apis mellifera foragers $(N=194)$ in the recall of olfactory memory $1 \mathrm{~h}$ after training to associate (-)-linalool, 1-octanol, or 2-octanone with sugar rewards. For each odour tested, honeybees were separated in three groups, with both antennae (white columns), only the right (gray columns), or only the left in use (black columns). 